\title{
Decision Procedure for Temporal Logic of Belief and Actions
}

\author{
Regimantas PLIUŠKEVIČIUS, Aida PLIUŠKEVIČIENÉ \\ Institute of Mathematics and Informatics \\ Akademijos 4, 08663 Vilnius, Lithuania \\ e-mail: \{regis,aida\}@ktl.mii.lt
}

Received: April 2004

\begin{abstract}
A temporal logic of belief and actions (TLBA) is considered. The TLBA allows us to express informational and dynamic properties of computational agents. The considered fragment of TLBA allows one: (1) to present a deduction-based structured decision procedure; (2) to separate a decision procedure for so-called induction-free formulas and (3) to use only logical axioms for such formulas. The main new technical tool of the presented decision procedure is separation rules which incorporate traditional rules for the temporal operator "next", belief modalities and action constants.
\end{abstract}

Key words: rational agent, temporal, modal, and dynamic logics, agent-based logic, deductionbased decision procedure, sequent calculus.

\section{Introduction}

Design of systems that are required to perform high-level management and control tasks in complex dynamic environments is becoming of increasing commercial importance. Such systems include the management and control of air traffic systems, telecommunications networks, business processes, space vehicles, medical services. It is now widely accepted that computational agents technology will have a key role to play in the development of the twenty-first century computer systems. The computational agents are often called as rational (or intelligent) agents because they make good decisions about what to do (Wooldridge, 2000; Wooldridge and Jennings, 1995).

There has recently been much interest in the use of mathematical logic for developing formal theories of such agents. Much of this interest arises from the fact that rational agents are increasingly being recognized as an important concept in computer science, software engineering, and artificial intelligence. It is recognized that logical proof methods are required for: (1) reasoning within the rational agents; (2) verification of agentbased systems with respect to the specification; (3) studying the properties of rational agents formalism.

One of the main tasks to solve these problems is to find decision procedures allowing us to tell in an automatic way whether the given specifications are provable (or true) in some logical formalism. A popular approach to formal methods in the investigation 
of properties of computer systems is concerned with model-checking (see, e.g., Clark et al., 2000). Unfortunately, the model-checking approach runs into serious difficulties when we consider complex agent-based computer systems. Therefore, along with the model-checking approach, a deductive approach (based on logical calculi) is widely used. Deduction-based decision procedures will not only tell us whether a given specification is provable (true) or not, but also give the proof of the specification whenever it is provable. The deductive approach is more promising in the case of first-order agent-based logics.

The best known logical theories of rational agents are $B D I$ logics (Rao and Georgeff, 1991 ) and $K A R O$ logics (Meyer and van der Hoek, 1995). In $B D I$ logics, each rational agent is viewed as having three mental attitudes: belief (the main component of $B D I$ logics), desire, and intention. The $B D I$ logics (for Belief, Desire, and Intention) are fusion of various propositional temporal logics and propositional multi-modal logics expressing the properties of the mental attitudes. The KARO logics (for Knowledge, Abilities, Results, and Opportunities) focus on the dynamics of mental states: how actions can change the agents knowledge (beliefs), desires, and so on. The $K A R O$ logics are a combination of propositional dynamic logic (Harel et al., 2000), logics of knowledge and commitment logics (Meyer and van der Hoek, 1995). The KARO logics do not have explicit temporal modalities and semantically are more complex than $B D I$ logics. In (Fisher and Wooldridge, 1997) it is presented a powerful concurrent METATEM system, based on the first-order temporal logic with multi-agent belief operators. In (Wooldridge, 2000) it is described a rich logic LORA (Logic of Rational Agents), based on a three-sorted first-order logic, $B D I$ logic, and a dynamic logic. Unfortunately, the MET ATEM and $L O R A$ are neither complete nor decidable. Decision procedures for $B D I$ logics, based on tableau-like calculi, are presented in (Rao and Georgeff, 1998). A different, more obvious, decision procedure (based on sequent-like calculus) for a branching $B D I$ logic is presented in (Nide and Tanaka, 2002). Tableau-based decision procedures for propositional temporal logics of knowledge and belief are described in (Wooldridge et al., 1998).

In this paper, a temporal logic of belief and action $(T L B A)$ is considered. The $T L B A$ is a fusion of the three logics: Computational Tree Logic $(C T L)$ (Emerson, 1990), multimodal logic $K D 45_{n}$ for belief modalities (Wooldridge, 2000), and propositional dynamic logic (Harel et al., 2000). Since the belief modalities are the main modalities of $B D I$ logics, the logic $T L B A$ can be considered as a core of the propositional fragment of the LORA logic (Wooldridge, 2000).

The $T L B A$ allows us to express informational and dynamic properties of rational agents. The aim of this paper is to present a deduction-based decision procedure for $T L B A$. The presented decision procedure is based on sequent-like calculus. For simplicity, we consider $R$-sequents of $T L B A$ (Section 3). The $R$-sequents of $T L B A$ allow us: 1 ) to present the proposed procedure in structured way; 2 ) to separate a decision procedure for so-called induction-free sequents and 3 ) to use only logical axioms (in leaves of derivations) for induction-free sequents. In the general case, we used, as in (Pliuškevičius, 1993, 1994, 1996, 1998, 2001; Nide and Tanaka, 2002; Pliuškevičius and Pliuškevičiené, 2003), non-logical axioms along with the logical ones. But these non-logical axioms are captured here in a simpler, more evident and more structured way. Moreover, since the 
$T L B A$ contains a propositional dynamic logic, the presented decision algorithm (in a special case) can be used as new decision algorithms for the multi-modal $K D 45_{n}$ and for the considered fragment of a propositional dynamic logic.

The paper is organized as follows. In Section 2, the components of the $T L B A$ are described. In Section 3, the language of the $T L B A$ is presented. In Section 4, the Gentzenlike decision procedure for a propositional logic is briefly recalled. In Section 5, auxiliary tools for the presented decision procedure are described. In Sections 6 and 7, decision algorithms for so-called induction-free $R$-sequents and arbitrary $R$-sequents are presented. In Section 8, conclusions and further investigations are briefly discussed.

\section{Components of $T L B A$}

The TLBA consists of three components: (1) logical, (2) informational and (3) dynamic.

The logical component of TLBA consists of the classical propositional logic with usual logical operators $\supset, \wedge, \vee, \neg$.

The informational component of TLBA consists of the multi-modal logic $K D 45_{n}$ (doxastic logic or weak- $S 5_{n}$ ) allowing us to represent an agent's beliefs and containing belief operators $\operatorname{Bel}(i), i \in\{1, \ldots, k\}(k \geqslant 1), i$ is a constant that stands for an agent. The semantics of multi-modal logics $K D 45_{n}$ (and other normal modal logics) is based on Hintikka-Kripke (or possible worlds) semantics using the notion of a reachability (accessibility) relation (Fitting, 1993). The Kripke structure consists of a non-empty set whose elements are called worlds and binary relations. These relations define which worlds are considered accessible from other world. As in (Wooldridge, 2000) we assume that the reachability relation of belief operators are distributive, transitive, serial, and Euclidean. Therefore the formulas $\operatorname{Bel}(i)(P \supset Q) \wedge \operatorname{Bel}(i) P \supset \operatorname{Bel}(i) Q, \operatorname{Bel}(i) P \supset$ $\operatorname{Bel}(i) \operatorname{Bel}(i) P, \operatorname{Bel}(i) P \supset \neg \operatorname{Bel}(i) \neg P$, and $\neg \operatorname{Bel}(i) P \supset \operatorname{Bel}(i) \neg \operatorname{Bel}(i) P$ (expressing, correspondingly, the distributive, transitive, serial and Euclidean properties of the reachability relation) are valid in $K D 45_{n}$. The multi-modal logic $K D 45_{n}$ allows us to compose belief modalities of different agents. This property permits us to express complex informational aspects about agent-based systems. Using the logic $K D 45_{n}$, we get information of the following form: agents have belief about themselves, other agents and the environment. The belief modalities are the main modalities in the theory and practice of rational agents (Wooldridge, 2000). Other, simpler modal operators are desire and intention operators (Wooldridge, 2000) satisfying only distributive and serial properties of the reachability relation.

The dynamic component of $T L B A$ consists of two parts: a temporal part and an agent part.

The temporal part of TLBA consists of a Computational Tree Logic $(C T L)$, often regarded as the simplest useful branching-time temporal logic (Emerson, 1990). The time is discrete and branching. The $C T L$ contains operators which operate the paths that are possible from a given state. In the linear-time temporal logic, operators are provided for describing events along a single computation path. The temporal component of $T L B A$ 
allows us to specify the temporal properties of an agent-based system: how the state of an agent and the environment changes over time.

The agent part of $T L B A$ consists of a propositional non-deterministic dynamic logic $(P D L)$ (Harel et al., 2000). Instead of elementary programs (as in the traditional $P D L$ ), we consider action constants, representing actions or actions sequences and interpreted as an arbitrary binary relation, and satisfying the simplest multi-modal logic $K_{n}$ (Fitting, 1993). The $P D L$ allows us to represent the actions (or actions sequences) that agents perform, and the effects of these actions. Using $P D L$ operators we can get composed actions. The main operation $*$ is often called a "star" operation and the $P D L$ without this operation is called a star-free $P D L$.

\section{Language of $T L B A$}

The language of $T L B A$ contains: a set of propositional symbols $P, P_{1}, P_{2}, \ldots, Q$, $Q_{1}, Q_{2}, \ldots$ (it is assumed that all propositional symbols are flexible, i.e., their values change in time); a set of agent constants $i, i_{1}, i_{2}, \ldots, j, j_{1}, j_{2}, \ldots, a_{1}, a_{2}, \ldots, b_{1}, b_{2}, \ldots$, $c_{1}, c_{2}, \ldots\left(i, j, i_{l}, j_{l}, a_{l}, b_{l}, c_{l} \in\{1, \ldots, k\}\right)$; a set of belief modalities $\operatorname{Bel}(i)(i \in$ $\{1, \ldots, k\}, k \geqslant 1$ ); temporal operators: $\bigcirc$ ("next"), $U$ ("until"), path quantifiers $\forall$ ("for each path"), $\exists$ ("there exists a path"); a set of action constants $\alpha_{1}, \alpha_{2}, \ldots, \beta_{1}, \beta_{2}, \ldots$; action constructors: ० (“composition"), $\cup$ ("non-deterministic choice"), * ("non-deterministic iteration" or "star"), ? ("test"); true (logical constant for truth); logical operators: $\supset$ (implication), $\wedge$ (conjunction), $\vee$ (disjunction), $\neg$ (negation).

Formulas and actions of $T L B A$ are defined inductively as follows: every propositional symbol and truth constant true are formulas; any action constant is an action; if $\alpha$ and $\beta$ are actions, then $(\alpha \circ \beta),(\alpha \cup \beta),\left(\alpha^{*}\right)$ are actions; if $A, B$ are formulas, then $A \supset B, A \wedge B, A \vee B, \neg(A)$ are formulas; if $P$ is a formula containing only propositional and logical symbols, then $P$ ? is an action; if $i$ is an agent constant, $A$ is a formula, then $\operatorname{Bel}(i) A$ is a formula; if $A, B$ are formulas, $Q$ is path quantifier $(Q \in\{\forall, \exists\})$, then $Q \bigcirc A$ and $Q(A U B)$ are formulas. The formula $A$ is a logical one if $A$ contains only logical and propositional symbols. Belief modalities can be nested. For example, formula $\operatorname{Bel}(i) \operatorname{Bel}(j) P$, where $P$ is a proposition "John is a good programmer", means "agent $i$ believes that agent $j$ believes that John is a good programmer".

The logical symbols $\supset, \wedge, \vee, \neg$ have their usual meaning. The operator $\bigcirc$ is the next time operator; the formula $\forall \bigcirc B(\exists \bigcirc B)$ intuitively means that $B$ is true in every (in some, respectively) immediate successor of the current state. It is clear that $\exists \bigcirc B=\neg \forall \bigcirc \neg B$ and $\forall \bigcirc B=\neg \exists \bigcirc \neg B$. The operator $U$ means until, the formula $\forall(A U B)(\exists(A U B))$ means that, for every path (for some path), $A$ is true until $B$ is true. The monadic modalities $\diamond$ ("now or some time in future") and $\square$ ("now and always in future") are defined using the modality "until": $Q \diamond A=Q($ true $U A), Q \in\{\forall, \exists\}$, $\forall \square A=\neg \exists \triangleright \neg A$. Since the invariant rules (see Section 7) for the temporal modality $\mathcal{W}$ ("unless") and action modality * ("star") have a similar shape, instead modality $U$, we shall use modality $\mathcal{W}$ ("unless" or "weak until"). The formula $\forall(A \mathcal{W} B)(\exists(A \mathcal{W} B))$ 
means that, for every path (for some path), either $A$ is true until $B$ is true, or else, $A$ is always true, i.e., $Q(A \mathcal{W} B)=Q(A U B) \vee Q \square A$; moreover, $Q \square A=Q(A \mathcal{W} \neg$ true $)$.

Therefore in formulas we consider three types of modalities, namely, temporal modalities: $\bigcirc$ and $\mathcal{W}$; belief modalities: $\operatorname{Bel}(i)$, and action modalities $\left[\alpha_{i}\right]$.

The path quantifier $\forall(\exists)$ expresses inevitability (optional, correspondingly) temporal properties. For example, the formula $\operatorname{Bel}(i) \forall\left(P_{1} \mathcal{W} P_{2}\right)$ means: "agent $i$ believes that inevitably $P_{1}$ is true until $P_{2}$ is true, or $P_{1}$ is inevitably true" and the formula $\operatorname{Bel}(i) \exists\left(P_{1} \mathcal{W} P_{2}\right)$ means: "agent $i$ believes that optionally $P_{1}$ is true until $P_{2}$ is true, or $P_{1}$ is optionally true".

Now, let us consider the action component of $T L B A$. The formula $[\alpha] A$ means: "every possible execution of the action $\alpha$ leads to a situation in which $A$ is true". The compound actions have the following meaning: $\alpha \circ \beta$ - "do $\alpha$ followed by $\beta$ "; $\alpha \cup \beta-$ "either $\alpha$ or $\beta$, non-deterministically"; $\alpha^{*}$ - "repeat $\alpha$ a finite, but non-deterministically"; $A$ ? - "proceed if $A$ true, else fail". The Algol-like program constructions "if $P$ then $\alpha$ else $\beta$ " and "while $P$ do $\alpha$ " can be expressed using the action operators as follows, $(P ? \circ \alpha) \cup(\neg P$ ? $\circ \beta)$ and $((P ? \circ \alpha) \cup(\neg P ?))^{*}$, respectively.

Together with other operators, the action operators allow us to express informational and dynamic properties of rational agents. For example, the formula $\forall \square \operatorname{Bel}(i)\left[\alpha^{*}\right] P$ (where $\alpha$ means the action "extract an important information from a company" and $P$ means "get a lot of money") means "inevitably always agent $i$ believes that, after repeated extraction of an important information from a company more than once he gets a lot of money".

The temporal operators and the action operator "star" allow us to express so-called induction-like properties. Namely:

$A \wedge Q \square(A \supset \bigcirc A) \supset Q \square A$ (induction-like property of the temporal operator "always"), $Q \in\{\forall, \exists\}$;

$C \wedge Q \square((C \wedge \neg B) \supset(A \wedge \bigcirc C)) \supset Q(A \mathcal{W} B)$ (induction-like property of the temporal operator "unless"), $Q \in\{\forall, \exists\}$;

$A \wedge\left[\alpha^{*}\right](A \supset[\alpha] A) \supset\left[\alpha^{*}\right] A$ (induction-like property of the action operator "star").

The first two operators express the main property of the temporal operators "always" and "unless"; the third one expresses the main property of the action operator "star".

The semantics of temporal operators $\square$ ("always"), $\mathcal{W}$ ("unless"), and the action operator * ("star") explicitly expresses the infinitary rules for these operators (see, e.g., Emerson, 1990; Kawai, 1987; Harel et al., 2000; Pliuškevičius, 1996).

$$
\begin{aligned}
& \frac{\left\{\Gamma \rightarrow \Delta, Q \bigcirc^{k} A\right\}_{k \in \omega}}{\Gamma \rightarrow \Delta, Q \square A}(\rightarrow \square \omega), \\
& \frac{\left\{\Gamma \rightarrow \Delta, \mathcal{W}_{k}\right\}_{k \in \omega}}{\Gamma \rightarrow \Delta, Q(A \mathcal{W} B)}\left(\rightarrow \mathcal{W}_{\omega}\right), \\
& \frac{\left\{\Gamma \rightarrow \Delta,[\alpha]^{k} A\right\}_{k \in \omega}}{\Gamma \rightarrow \Delta,\left[\alpha^{*}\right] A}\left(\rightarrow *_{\omega}\right),
\end{aligned}
$$


where $\omega=\{0,1, \ldots\} ; \bigcirc^{k} A=A$ (if $k=0$ ), $\bigcirc^{k} A=\bigcirc \bigcirc^{k-1} A$ (if $k \geqslant 1$ ); $\mathcal{W}_{0}=$ $A \vee B ; \mathcal{W}_{k}=B \vee\left(A \wedge Q \bigcirc \mathcal{W}_{k-1}\right)(k \geqslant 1) ;[\alpha]^{k} A=A$ (if $\left.k=0\right),[\alpha]^{k} A=[\alpha]\left[\alpha^{k-1}\right] A$ (if $k \geqslant 1$ ); $Q \in\{\forall, \exists\}$.

Infinitary rules are widely used in the logics of programs and agent-based logics.

Instead of formulas we consider sequents, i.e., formal expressions $A_{1}, \ldots, A_{n} \rightarrow$ $B_{1}, \ldots, B_{m}$ (where $A_{1}, \ldots, A_{n}\left(B_{1}, \ldots, B_{m}\right)$ is a set of formulas) which are interpreted as the formula $\wedge_{i=1}^{n} A_{i} \supset \vee_{j=1}^{m} B_{j}, i, j \geqslant 0$.

To describe the considered fragment of $T L B A$, let us recall the notions of positive and negative occurrences (see, e.g., Wang, 1964).

A formula (or some symbol) occurs positively in some formula $B$ if it appears within the scope of no negation sign or in the scope of an even number of the negation sign, once all the occurrences of $A \supset C$ have been replaced by $\neg A \vee C$; in the opposite case, the formula (symbol) occurs negatively in $B$. For a sequent $S=A_{1}, \ldots, A_{n} \rightarrow B_{1}, \ldots, B_{m}$ positive and negative occurrences are determined just like for the formula $\wedge_{i=1}^{n} A_{i} \supset$ $\vee_{i=1}^{m} B_{i}$.

EXAMPLE 1. Let $S=\forall(A \mathcal{W}(B \vee C)) \rightarrow \exists(A \mathcal{W} B), \exists(A \mathcal{W} C)$, then the first (from the left) occurrence of the symbol $\mathcal{W}$ and the occurrences of the symbols $\forall$ and $\vee$ are negative, two second occurrences of the symbol $\mathcal{W}$ and two occurrences of the symbol $\exists$ are positive.

A sequent $S$ is an $R$-sequent, if $S$ satisfies the following regularity condition: if formulas $Q(A \mathcal{W} B)(Q \in\{\forall, \exists\})$ and/or $\left[\alpha^{*}\right] A$ occur negatively in $S$, then $A$ and $B$ do not contain positive occurrences of the temporal operator $\mathcal{W}$ and positive occurrences of the action operator * ("star"), and action constants.

EXAMPLE 2. Let $S_{1}$ be a sequent obtained from the sequent $S$ (Example 1), taking instead of $A, B, C$ the formulas $\operatorname{Bel}(i), \neg \operatorname{Bel}(j)$, and $\left[\alpha_{1}^{*}\right] P$, respectively; let $S_{2}$ be a sequent obtained from the sequent $S$ (Example 1), taking instead of $A, B, C$ the formulas $\neg \forall(P \mathcal{W} Q), \neg \operatorname{Bel}(j)$ and $\neg\left[\alpha_{1}^{*}\right] P$, correspondingly. Then the sequent $S_{1}$ is an $R$-sequent, but the sequent $S_{2}$ is not an $R$-sequent.

\section{Gentzen-like Decision Algorithm for a Propositional Logic}

Gentzen has applied his sequent calculi in the decision problem to the classical and intuitionistic propositional logic (Gentzen, 1934-35). The Gentzen algorithm was based on his main theorem on normal form derivations. According to this theorem, all the sequents in the derivation of a given sequent $S$ contain only some parts (subformulas) from the sequent $S$, i.e., during the construction of derivations nothing new can be obtained. Therefore bottom-up applying the rules of sequent calculus, the derivation must be terminated. Gentzen's decision algorithm was improved in (Wang, 1964), exploiting a notion of the invertible rule.

The rule $(i)$ is called invertible in a sequent calculus $I$, if the derivation in $I$ of the conclusion of $(i)$ implies the derivability in $I$ of each premise of $(i)$. Let $(i)$ be any rule. 
Usually $(i)$ is applied to get the conclusion of $(i)$ from the premises of $(i)$. Sometimes it is convenient to apply $(i)$ to get premises of $(i)$ from the conclusion of $(i)$. In this case, instead of "application of $(i)$ " we have a "bottom-up application of $(i)$ ". In case $(i)$ is invertible, the bottom-up application of $(i)$ preserves the derivability.

Definition 1 (calculus $G W$ ). The calculus $G W$ is defined by the following postulates. Axiom: $\Gamma, A \rightarrow \Delta, A$.

The formula $A$ is called the main formula of the axiom.

The rules consist of traditional invertible rules of inference:

$$
\begin{array}{ll}
\frac{\Gamma, A \rightarrow \Delta, B}{\Gamma \rightarrow \Delta,(A \supset B)}(\rightarrow \supset), & \frac{\Gamma \rightarrow \Delta, A ; \Gamma, B \rightarrow \Delta}{\Gamma,(A \supset B) \rightarrow \Delta}(\supset \rightarrow), \\
\frac{\Gamma \rightarrow \Delta, A ; \Gamma \rightarrow \Delta, B}{\Gamma \rightarrow \Delta,(A \wedge B)}(\rightarrow \wedge), & \frac{\Gamma, A, B \rightarrow \Delta}{\Gamma,(A \wedge B) \rightarrow \Delta}(\wedge \rightarrow), \\
\frac{\Gamma \rightarrow \Delta, A, B}{\Gamma \rightarrow \Delta,(A \vee B)}(\rightarrow \vee), & \frac{\Gamma, A \rightarrow \Delta ; \Gamma, B \rightarrow \Delta}{\Gamma,(A \vee B) \rightarrow \Delta}(\vee \rightarrow), \\
\frac{\Gamma, A \rightarrow \Delta}{\Gamma \rightarrow \Delta, \neg A}(\rightarrow \neg), & \frac{\Gamma \rightarrow \Delta, A}{\Gamma, \neg A \rightarrow \Delta}(\neg \rightarrow) .
\end{array}
$$

From the shape of the rules of the calculus we can see that each premise of some rule $(i)$ has less complexity (defined as the number of occurrences of logical symbols) than the conclusion of the rule $(i)$. Using this property, we can eliminate logical symbols (elimination property of the calculus $G W$ ). Using the elimination property of the calculus $G W$ starting from a given sequent $S$, we bottom-up apply the rules of the calculus $G W$ until in each branch we get an axiom or an "atomic" axiom (having propositional symbols as the main formulas of the axiom). In this case, $G W \vdash S$ (i.e., the sequent is derivable in the calculus $G W$ ), or there exists a branch, a leaf of which contains no axioms. By invertibility of the rules of calculus $G W$, in this case $G W \nvdash S$. Therefore the termination criterion of the decision procedure is rather simple: either in all the leaves we get axioms (or atomic axioms) - positive criterion, or in a leaf of some branch we get a sequent which is not an axiom.

Using induction on the hight of derivation, we can show (see, e.g., Pliuškevičius, 1990) that all the rules of the calculus $G W$ are invertible.

EXAMPLE 3. (a) Let $S=A \vee B \rightarrow(B \vee A) \wedge(B \vee \neg B)$, then by bottom-up applying (from left to right) the rule $(\vee \rightarrow)$ and afterwards the rules $(\rightarrow \wedge),(\rightarrow \vee),(\rightarrow \neg)$, we get four sequents $S_{1}=A \rightarrow B, A ; S_{2}=A, B \rightarrow B ; S_{3}=B \rightarrow B, A ; S_{4}=B, B \rightarrow B$, which are axioms. Therefore the procedure terminates positively and we get $G W \vdash S$.

(b) Let $S=A \vee B \rightarrow A \wedge(B \vee \neg B)$, then by bottom-up applying (from left to right) the rule $(\vee \rightarrow)$ and afterwards the rules $(\rightarrow \wedge),(\rightarrow \vee),(\rightarrow \neg)$, we get four sequents $S_{1}=A \rightarrow A ; S_{2}=A, B \rightarrow B ; S_{3}=B \rightarrow A ; S_{4}=B, B \rightarrow B$. The sequents $S_{1}$, $S_{2}, S_{4}$ are axioms, but $S_{3}$ is not an axiom. Therefore the process terminates negatively and we get $G W \nvdash S$. 


\section{Some Auxiliary Tools of the Decision Algorithm for $\boldsymbol{R}$-sequents}

The decision algorithm presented here is a substantial extension of the Gentzen-Wang algorithm, presented in the previous section. In this section, we present the main auxiliary tools of the decision algorithm for $R$-sequents: separation and reduction rules, and marked contraction rules. First we introduce some canonical forms of $R$-sequents.

An $R$-sequent $S$ is a primary $R$-sequent, if $S=\Sigma_{1}, \mathcal{B} \Gamma, \forall \bigcirc \Pi_{1}, \forall \mathcal{W} \Delta_{1},\left[\alpha_{q}\right] \Omega$, $\left[\alpha^{*}\right] \Theta_{1} \rightarrow \Sigma_{2}, \mathcal{B} \Delta, \forall \bigcirc \Pi_{2}, \forall \mathcal{W} \Delta_{2},\left[\beta_{k}\right] \nabla,\left[\alpha^{*}\right] \Theta_{2}$, where for every $i(i \in\{1,2\}) \Sigma_{i}$ is empty or consists of logical formulas; $\mathcal{B} \Gamma(\mathcal{B} \Delta)$ is empty or consists of formulas of the shape $\operatorname{Bel}\left(a_{k}\right) A, 1 \leqslant k \leqslant n\left(\operatorname{Bel}\left(b_{j}\right) B, 1 \leqslant j \leqslant m\right.$, correspondingly); $\forall \bigcirc \Pi_{i}$ is empty or consists of formulas of the shape $\forall \bigcirc A ; \forall \mathcal{W} \Delta_{i}$ is empty or consists of formulas of the shape $\forall(A \mathcal{W} B) ;\left[\alpha_{q}\right] \Omega\left(\left[\beta_{k}\right] \nabla\right)$ is empty or consists of formulas of the shape $\left[\alpha_{q}\right] A\left(\left[\beta_{k}\right] M\right.$, correspondingly), where $\alpha_{q}$ and $\beta_{k}$ are action constants; $\left[\alpha^{*}\right] \Theta_{i}$ is empty or consists of formulas of the shape $\left[\alpha^{*}\right] A$, where $\alpha$ is an action. An $R$-sequent $S$ is a reduced primary sequent if $S$ is a primary one not containing $\forall \mathcal{W} \Delta_{i},\left[\alpha^{*}\right] \Theta_{i}$, but $\Gamma, \Delta, \Pi_{i}, \Omega, \nabla$ may contain temporal operator $\mathcal{W}$ and action operator $*$.

Let us define reduction rules by means of which each $R$-sequent can be reduced to a set of primary and reduced primary $R$-sequents.

DEFINITION 2 (reduction rules). Reduction rules consist of the following rules:

- logical rules: all the rules of the calculus $G W$;

- temporal rules:

$$
\begin{aligned}
& \frac{\Gamma, \forall \bigcirc \neg A \rightarrow \Delta}{\Gamma \rightarrow \Delta, \exists \bigcirc A}(\rightarrow \exists \bigcirc), \quad \frac{\Gamma \rightarrow \Delta, \forall \bigcirc \neg A}{\exists \bigcirc A, \Gamma \rightarrow \Delta}(\exists \bigcirc \rightarrow), \\
& \frac{A \vee B, B \vee Q \bigcirc(Q(A \mathcal{W} B)), \Gamma_{1} \rightarrow \Delta_{1}}{Q(A \mathcal{W} B), \Gamma_{1} \rightarrow \Delta_{1}}(Q \mathcal{W} \rightarrow),
\end{aligned}
$$

where $\Gamma_{1} \rightarrow \Delta_{1}$ contains positive occurrences of $\mathcal{W}$ ("unless") or $\bigcirc$ ("next"),

$$
\frac{\Gamma \rightarrow \Delta, A, B ; \Gamma \rightarrow \Delta, B, Q \bigcirc(Q(A \mathcal{W} B))}{\Gamma \rightarrow \Delta, Q(A \mathcal{W} B)}(\rightarrow Q \mathcal{W}),
$$

where $Q \in\{\forall, \exists\}$;

$$
\frac{A \vee B, \Pi \rightarrow \Theta}{Q(A \mathcal{W} B), \Pi \rightarrow \Theta}\left(\mathcal{W}_{0} \rightarrow\right)
$$

where $\Pi \rightarrow \Theta$ does not contain positive occurrences either $\mathcal{W}$ ("unless") or $\bigcirc$ ("next");

- action rules:

$$
\frac{\Gamma \rightarrow \Delta,[\alpha][\beta] A}{\Gamma \rightarrow \Delta,[\alpha \circ \beta] A}(\rightarrow \circ), \quad \frac{[\alpha][\beta] A, \Gamma \rightarrow \Delta}{[\alpha \circ \beta] A, \Gamma \rightarrow \Delta}(\circ \rightarrow),
$$




$$
\begin{array}{ll}
\frac{\Gamma \rightarrow \Delta,[\alpha] A ; \Gamma \rightarrow \Delta,[\beta] A}{\Gamma \rightarrow \Delta,[\alpha \cup \beta] A}(\rightarrow \cup), & \frac{[\alpha] A,[\beta] A, \Gamma \rightarrow \Delta}{[\alpha \cup \beta] A, \Gamma \rightarrow \Delta}(\cup \rightarrow), \\
\frac{\Gamma, P \rightarrow \Delta, B}{\Gamma \rightarrow \Delta,[P ?] B}(\rightarrow ?), & \frac{\Gamma \rightarrow \Delta, P ; B, \Gamma \rightarrow \Delta}{[P ?] B, \Gamma \rightarrow \Delta}(? \rightarrow), \\
\frac{\Gamma \rightarrow \Delta, A ; \Gamma \rightarrow \Delta,[\alpha]\left[\alpha^{*}\right] A}{\Gamma \rightarrow \Delta,\left[\alpha^{*}\right] A}(\rightarrow *), & \frac{A,[\alpha]\left[\alpha^{*}\right] A, \Gamma_{1} \rightarrow \Delta_{1}}{\left[\alpha^{*}\right] A, \Gamma_{1} \rightarrow \Delta_{1}}(* \rightarrow),
\end{array}
$$

where $\Gamma_{1} \rightarrow \Delta_{1}$ contains positive occurrences of $*$ ("star") or action constants,

$$
\frac{A, \Pi \rightarrow \Theta}{\left[\alpha^{*}\right] A, \Pi \rightarrow \Theta}\left(*_{0} \rightarrow\right),
$$

where $\Pi \rightarrow \Theta$ does not contain positive occurrences either $*$ ("star") or action constants.

REMARK 1. (a) The temporal rules $(\rightarrow \exists \bigcirc),(\exists \bigcirc \rightarrow)$ correspond to the semantic equivalent between the path quantifiers: $\exists \bigcirc A \equiv \neg \forall \bigcirc \neg A$. The temporal rules $(Q \mathcal{W} \rightarrow),(\rightarrow Q \mathcal{W})$ correspond to the following semantic equivalent (see, e.g., Emerson, 1990) $(A \vee B) \wedge(B \vee Q \bigcirc(Q(A \mathcal{W} B))) \equiv Q(A \mathcal{W} B)$ (where $Q \in\{\forall, \exists\})$, and to the sequential rules for the "unless" operator from (Nide and Tanaka, 2002). The action rules $(\rightarrow *),(* \rightarrow)$ correspond to the semantic equivalent $\left[\alpha^{*}\right] A \equiv A \wedge[\alpha]\left[\alpha^{*}\right] A$ (see, e.g., Harel et al., 2000). The other action rules $(\rightarrow \circ),(\circ \rightarrow),(\rightarrow \cup),(\cup \rightarrow),(\rightarrow ?),(? \rightarrow)$ correspond (respectively) to the following semantic equivalent $[\alpha \circ \beta] A \equiv[\alpha][\beta] A$; $[\alpha \cup \beta] A \equiv[\alpha] A \wedge[\beta] A ;[P$ ? $] A \equiv P \supset A$ (see, e.g., Harel et al., 2000). The temporal rule $\left(\mathcal{W}_{0} \rightarrow\right)$ (action rule $\left(*_{0} \rightarrow\right)$ ) corresponds to the case of the infinitary rule $\left(\rightarrow \mathcal{W}_{\omega}\right)$ (rule $\left(\rightarrow *_{\omega}\right)$, respectively, when $k=0$ ) (see Section 3).

(b) The bottom-up applications of the temporal rule $(\rightarrow \exists \bigcirc),(\exists \bigcirc \rightarrow)$ eliminate occurrences of the path quantifier $\exists$. The bottom-up applications of the rule $\left(\mathcal{W}_{0} \rightarrow\right)$ (rule $\left(*_{0} \rightarrow\right)$ ) eliminate occurrences of the operator "unless" " "star", correspondingly).

We can see the same effect in the left premise of the rules $(\rightarrow Q \mathcal{W}),(\rightarrow *)$. The bottom-up applications of the action rules $(\rightarrow \circ),(\circ \rightarrow),(\rightarrow \cup),(\cup \rightarrow),(\rightarrow ?),(? \rightarrow)$ eliminate the occurrences of the action operators $\circ, \cup$, ?, correspondingly.

From the shape of the primary $R$-sequent it is easy to see that bottom-up applying logical rules, the rules $(\exists \bigcirc \rightarrow),(\rightarrow \exists \bigcirc)$ and action rules, except the rules for of the "star" operator, each $R$-sequent can be reduced to a set of primary $R$-sequents. As follows from the shape of reduced primary $R$-sequents bottom-up applying reduction rules each primary $R$-sequent can be reduced to a set of reduced primary $R$-sequents. For each reduced primary $R$-sequent the separation rules (see below) are bottom-up applied.

EXAmPle 4. Let $S=\left[\alpha_{1}^{*} \cup \alpha_{2}^{*}\right] P_{1}, \exists \bigcirc P_{2},\left(\operatorname{Bel}(i) P_{3} \vee \operatorname{Bel}(j) P_{4}\right) \rightarrow\left(\operatorname{Bel}(i) P_{5} \vee\right.$ $\left.\operatorname{Bel}(j) P_{6}\right),\left(P_{7} \supset\left[\alpha_{3}\right] P_{8}\right)$. Bottom-up applying logical rules $(\vee \rightarrow),(\rightarrow \vee),(\rightarrow \supset)$ and the rules $(\exists \circ \rightarrow),(\cup \rightarrow)$, we get two primary $R$-sequents: $S_{1}=\left[\alpha_{1}^{*}\right] P_{1}$, 
$\left[\alpha_{2}^{*}\right] P_{1}, \operatorname{Bel}(i) P_{3}, P_{7} \rightarrow \forall \bigcirc \neg P_{2}, \operatorname{Bel}(i) P_{5}, \operatorname{Bel}(j) P_{6},\left[\alpha_{3}\right] P_{8}$ and $S_{2}=\left[\alpha_{1}^{*}\right] P_{1}$, $\left[\alpha_{2}^{*}\right] P_{1}, \operatorname{Bel}(j) P_{4}, P_{7} \rightarrow \forall \bigcirc \neg P_{2}, \operatorname{Bel}(i) P_{5}, \operatorname{Bel}(j) P_{6},\left[\alpha_{3}\right] P_{8}$. Bottom-up applying the rule $(* \rightarrow)$ to $S_{1}, S_{2}$ we get two reduced primary $R$-sequents $S_{1}^{*}=$ $P_{1},\left[\alpha_{1}\right]\left[\alpha_{1}^{*}\right] P_{1}, P_{1},\left[\alpha_{2}\right]\left[\alpha_{2}^{*}\right] P_{1}, \operatorname{Bel}(i) P_{3}, P_{7} \rightarrow \forall \bigcirc \neg P_{2}, \operatorname{Bel}(i) P_{5}, \operatorname{Bel}(j) P_{6},\left[\alpha_{3}\right] P_{8}$ and $S_{2}^{*}=P_{1},\left[\alpha_{1}\right]\left[\alpha_{1}^{*}\right] P_{1}, P_{1},\left[\alpha_{2}\right]\left[\alpha_{2}^{*}\right] P_{1}, \operatorname{Bel}(j) P_{4}, P_{7} \rightarrow \forall O \neg P_{2}, \operatorname{Bel}(i) P_{5}$, $\operatorname{Bel}(j) P_{6},\left[\alpha_{3}\right] P_{8}$.

As pointed out in (Hudelmaier, 1996), one of rather effective techniques (instead of traditional ones) for deciding the provability of sequents in $S 4_{n}$ is based on loop checking (the same techniques is also used for $K D 45_{n}$ ). Namely, if "essentially the same" sequent occurs twice on a branch of a constructed deduction, then there is a shorter deduction with the same end sequent which does not show this redundancy, and one may backtrack. This loop checking requires quite involved implementation techniques. Therefore, contrary to loop checking, we propose a so-called loop exclusion (for belief modalities) method. The loop exclusion corresponds to the construction of a contraction-free sequent calculus (see, e.g., Hudelmaier, 1996).

First, let us introduce the simple separation rules.

DEFINITION 3 (separation rules $\left(S R_{l}^{*}\right)$ ). The separation rules $\left(S R_{l}^{*}\right)$ are of the following shape:

$$
\frac{S_{l}}{\Sigma_{1}, \mathcal{B} \Gamma, \forall \bigcirc \Pi_{1},\left[\alpha_{q}\right] \Omega \rightarrow \Sigma_{2}, \mathcal{B} \Delta, \forall \bigcirc \Pi_{2},\left[\beta_{k}\right] \nabla}\left(S R_{l}^{*}\right),
$$

where $1 \leqslant l \leqslant 3$, the conclusion of the rules $\left(S R_{l}^{*}\right)$ is a reduced primary $R$-sequent, such that $G W \nvdash \Sigma_{1} \rightarrow \Sigma_{2}$.

Let $\forall \bigcirc \Pi_{2}=\forall \bigcirc A_{1}, \ldots, \forall \bigcirc A_{n}(n \geqslant 1)$ or $\forall \bigcirc \Pi_{2}=\varnothing$, then

$S_{1}=\Pi_{1} \rightarrow A_{i}^{0}(1 \leqslant i \leqslant n)$, where $A_{i}^{0} \in\left\{\varnothing, A_{i}\right\}$ and $A_{i}^{0}=\varnothing$ if $\forall \bigcirc \Pi_{2}=\varnothing$, and $A_{i}^{0}=A_{i}$ if $\forall \bigcirc \Pi_{2} \neq \varnothing$.

Let $\left[\beta_{k}\right] \nabla=\left[\beta_{1}\right] \nabla_{1}, \ldots,\left[\beta_{n}\right] \nabla_{n}(n \geqslant 1) ;\left[\alpha_{q}\right] \Omega=\left[\alpha_{1}\right] \Omega_{1}, \ldots,\left[\alpha_{m}\right] \Omega_{m}(m \geqslant 0)$, where $\left[\alpha_{j}\right] \Omega_{j}, 0 \leqslant j \leqslant m\left(\left[\beta_{i}\right] \nabla_{i}, 1 \leqslant i \leqslant n\right)$ consists of formulas of the shape $\left[\alpha_{j}\right] B_{\rho, j}$ (of the shape $\left[\beta_{i}\right] M_{\rho, i}$, respectively), moreover, $\left[\alpha_{j}\right] \Omega_{j}$ may be the empty word, then

$S_{2}=\Omega_{j}^{0} \rightarrow M_{\rho, i}(1 \leqslant i \leqslant n, 1 \leqslant j \leqslant m, \rho \geqslant 1)$, where $\Omega_{j}^{0}=\Omega_{j}$, if $\alpha_{j}=\beta_{i}$ and $\Omega_{j}^{0}=\varnothing$, if $\alpha_{j} \neq \beta_{i}$ or $\left[\alpha_{q}\right] \Omega=\varnothing$.

$S_{3}=\mathcal{B} \Gamma_{m}, \Gamma_{m} \rightarrow \mathcal{B} \Delta_{n}, A^{0}, \operatorname{Bel}(k) A^{0}$, where $A^{0} \in\{\varnothing, A\}, \mathcal{B} \Delta_{n}, \operatorname{Bel}(k) A^{0} \subseteq$ $\mathcal{B} \Delta, \mathcal{B} \Gamma_{m} \subseteq \mathcal{B} \Gamma$, and $\mathcal{B} \Gamma_{m}\left(\mathcal{B} \Delta_{n}\right)$ is empty or consists of the formulas of the shape $\operatorname{Bel}(k) M$.

The rule $\left(S R_{3}^{*}\right)$ (which corresponds non-invertible rule of Shwart's (1989)) does not reflect peculiarities of belief modalities, i.e., distributivity, transitivity, seriality, and Euclidean properties, and it is directed to the realization of the loop checking method. Therefore we shall use more sophisticated separation rules for belief modalities, which reflect peculiarities of belief modalities and are directed to the realization of the loop exclusive method (for induction-free $R$-sequents, see below). 
To define the new separation rules let us introduce two sorts of marks of belieftype modalities, namely, the first sort of marks is of the shape $\sigma^{*}$ (where $\sigma \in$ $\left.\left\{\operatorname{Bel}(i), \bigcirc, \mathcal{W},\left[\alpha_{k}\right]\right\}\right)$, and the second sort of marks has the shape $\mathrm{Bel}^{+}(i)$. The first sort of marks is defined as follows: if $B=B e l^{*}(i) A$, then each occurrence of belief, temporal and action modalities in $A$ is marked by the first sort of marks and $\sigma^{* *}=\sigma^{*}$. The second sort of marks is used only for positive occurrences of belief modalities in the sequent and only in formulas of the shape $\operatorname{Bel}(i) A$. The first sort of marks is used for positive and negative occurrences of modalities. Both of these marks are meant to restrict applications of new separation rules for belief modalities and to exclude loops for induction-free $R$-sequents.

DEFINITION 4 (separation rules $\left(S R_{l}\right)$ ). The separation rules $\left(S R_{l}\right)(1 \leqslant l \leqslant 5)$ are obtained from the rules $\left(S R_{l}^{*}\right)(1 \leqslant l \leqslant 3)$ replacing the rule $\left(S R_{3}^{*}\right)$ by the rules $\left(S R_{3}\right),\left(S R_{4}\right)$ and $\left(S R_{5}\right)$ having the same conclusion as the rule $\left(S R_{3}^{*}\right)$ and the following premises.

Let $\operatorname{Bel}(l) A_{\rho, l} \in \mathcal{B} \Delta$, where $A_{\rho, l}$ contains at least one negative occurrence of a belief modality and $\operatorname{Bel}(l) A_{\rho, l}(1 \leqslant l \leqslant p, \rho \geqslant 1)$ has no second sort of marks, $\mathcal{B} \Delta^{\prime}=\mathcal{B} \Delta \backslash \operatorname{Bel}(l) A_{\rho, l}$, where $\mathcal{B} \Delta^{\prime}=\operatorname{Bel}\left(b_{1}\right) \Delta_{1}, \ldots, \operatorname{Bel}\left(b_{m}\right) \Delta_{m}(m \geqslant 0)$; $\mathcal{B} \Gamma=\operatorname{Bel}\left(a_{1}\right) \Gamma_{1}, \ldots, \operatorname{Bel}\left(a_{n}\right) \Gamma_{n}(n \geqslant 0)$, where $\operatorname{Bel}\left(a_{k}\right) \Gamma_{k}, 0 \leqslant k \leqslant n\left(\operatorname{Bel}\left(b_{j}\right) \Delta_{j}\right.$, $0 \leqslant j \leqslant m$ ) is empty or consists of formulas of the shape $\operatorname{Bel}\left(a_{k}\right) M$ (of the shape $\operatorname{Bel}\left(b_{j}\right) \mathrm{B}$, respectively). Then

$S_{3}=B e l^{*}\left(a_{k}\right) \Gamma_{k}^{0}, \Gamma_{k}^{0} \rightarrow B e l^{*}\left(b_{j}\right) \Delta_{j}^{0 \prime}, A_{\rho, l}, \mathrm{Bel}^{+}(l) A_{\rho, l}$, where $B e l^{*}\left(a_{k}\right) \Gamma_{k}^{0}=\varnothing$, $\Gamma_{k}^{0}=\varnothing\left(B e l^{*}\left(b_{j}\right) \Delta_{j}^{0 \prime}=\varnothing\right)$, if $a_{k} \neq l\left(b_{j} \neq l\right)$ and $B e l^{*}\left(a_{k}\right) \Gamma_{k}^{0}=B e l^{*}\left(a_{k}\right) \Gamma_{k}$ $\left(B e l^{*}\left(b_{j}\right) \Delta_{j}^{0 \prime}=B e l^{*}\left(b_{j}\right) \Delta_{j}^{\prime}\right)$, in the opposite case.

Let $\operatorname{Bel}(l) A_{\rho, l} \in \mathcal{B} \Delta(1 \leqslant l \leqslant p, \rho \geqslant 1)$, and $\operatorname{Bel}(l) A_{\rho, l}$ has no second sort of marks, and $\mathcal{B} \Gamma=\operatorname{Bel}\left(a_{1}\right) \Gamma_{1}, \ldots, \operatorname{Bel}\left(a_{n}\right) \Gamma_{n}(n \geqslant 0)$, where $\operatorname{Bel}\left(a_{k}\right) \Gamma_{k}(0 \leqslant k \leqslant n)$ is empty or consists of formulas of the shape $\operatorname{Bel}\left(a_{k}\right) M$. Then

$S_{4}=B e l^{*}\left(a_{k}\right) \Gamma_{k}^{0}, \Gamma_{k}^{0} \rightarrow A_{\rho, l}, \mathrm{Bel}^{+}(l) A_{\rho, l}$, where $\Gamma_{k}^{0}=\Gamma_{k}$, if $a_{k}=l$, and $\Gamma_{k}^{0}=$ $\varnothing, \mathrm{Bel}^{*}\left(a_{k}\right) \Gamma_{k}=\varnothing$ in the opposite case; moreover, $\operatorname{Bel}^{*}\left(a_{k}\right) \Gamma_{k}=\varnothing$, if $\operatorname{Bel}(l) A_{\rho, l}$ and each formula from $\operatorname{Bel}\left(a_{k}\right) \Gamma_{k}$ contains the same number of occurrences (different polarity) of the same belief modalities.

Let $\mathcal{B} \Delta$ is empty or consists of arbitrary formulas of the shape $\operatorname{Bel}(l) A$ and $\mathcal{B} \Gamma$ be of the same shape as in the previous case, then

$S_{5}=\operatorname{Bel}^{*}\left(a_{k}\right) \Gamma_{k}^{0}, \Gamma_{k} \rightarrow$, where $\operatorname{Bel}^{*}\left(a_{k}\right) \Gamma_{k}^{0}=\varnothing$, if $\Gamma_{k}=\operatorname{Bel}\left(c_{1}\right), \ldots$, $\operatorname{Bel}\left(c_{q}\right) \Gamma_{k}^{\prime}(q \geqslant 0)$ and $\Gamma_{k}^{\prime}$ does not contain positive occurrences of belief modalities, and $B e l^{*}\left(a_{k}\right) \Gamma_{k}^{0}=B e l^{*}\left(a_{k}\right) \Gamma_{k}$, in the opposite case.

REMARK 2. (a) Using peculiarities of the belief modalities it is easy to prove that each application of the rule $\left(S R_{3}^{*}\right)$ can be replaced by the applications of the rules $\left(S R_{3}\right)$, $\left(S R_{4}\right),\left(S R_{5}\right)$, and vice versa.

(b) The rule $\left(S R_{3}\right)$ corresponds to the Euclidean property of belief modalities. The rule $\left(S R_{4}\right)$ corresponds to distributivity, transitivity, and seriality properties of belief modalities. The rule $\left(S R_{5}\right)$ corresponds to the seriality property of belief modalities.

(c) The separation rules $\left(S R_{l}\right)(1 \leqslant l \leqslant 5)$ incorporate the following rules: 
- the rule for the "next" operator:

$$
\frac{\Pi \rightarrow A^{0}}{\forall \bigcirc \Pi \rightarrow \forall \bigcirc A^{0}}(\forall \bigcirc),
$$

where $\forall \bigcirc \Pi$ is empty or consists of formulas of the shape $\forall \bigcirc B ; A^{0} \in\{\varnothing, A\}$;

- the rule for the action constants:

$$
\frac{\Omega \rightarrow A}{\left[\alpha_{i}\right] \Omega \rightarrow\left[\alpha_{i}\right] A}\left[\alpha_{i}\right]
$$

where $\left[\alpha_{i}\right] \Omega$ is empty or consists of formulas of the shape $\left[\alpha_{i}\right] B$;

- the non-invertible rules for belief modalities (which incorporate the rules for belief modality (Nide and Tanaka, 2000)):

$$
\begin{aligned}
& \frac{\operatorname{Bel}(i) \Gamma, \Gamma \rightarrow \operatorname{Bel}(i) \Delta, A^{0}, \operatorname{Bel}(i) A^{0}}{\operatorname{Bel}(i) \Gamma \rightarrow \operatorname{Bel}(i) \Delta, \operatorname{Bel}(i) A^{0}}(\operatorname{Bel}-K D 45), \\
& \frac{\Gamma \rightarrow A}{\operatorname{Bel}(i) \Gamma \rightarrow \operatorname{Bel}(i) A}(\operatorname{Bel}-K),
\end{aligned}
$$

where $A^{0} \in\{\varnothing, A\}$ and $\operatorname{Bel}(i) A^{0}=\varnothing$ if $A^{0}=\varnothing$, and $\operatorname{Bel}(i) A^{0}=\operatorname{Bel}(i) A$, if $A^{0}=A ; \operatorname{Bel}(i) \Gamma(\operatorname{Bel}(i) \Delta)$ is empty or consists of formulas of the shape $\operatorname{Bel}(i) M$; the rule (Bel-KD45) corresponds to the modal logic KD45 and the rule $(B e l-K)$ to the modal logic $K$;

- the structural rule of weakening:

$$
\frac{\Theta_{1} \rightarrow \Theta_{2}}{\Delta_{1}, \Theta_{1} \rightarrow \Delta_{2}, \Theta_{2}}(W)
$$

DEFINITION 5 (marked contraction rules). During the reduction to primary and reduced primary $R$-sequents the marked contraction rules $\mathrm{Bel}^{+}(i) A, \mathrm{Bel}^{\circ}(i) A=\mathrm{Bel}^{+}(i) A$ (where $\circ \in\{\varnothing, *\}$ ) and $\sigma^{*} A, \sigma A=\sigma^{*} A$ (where $\sigma \in\left\{\operatorname{Bel}(i), \bigcirc, \mathcal{W},\left[\alpha_{k}\right]\right\}$ ) and the ordinary contraction rule $A, A=A$ (which follows from the set-type notion of a sequent) will be used implicitly.

REMARK 3. (a) A bottom-up application of the separation rule $\left(S R_{1}\right)$ (rule $\left(S R_{2}\right)$ ) eliminates occurrences of the symbol $\bigcirc$ ("next") (the occurrences of action constants, correspondingly). The rules $\left(S R_{3}\right),\left(S R_{4}\right)$ and $\left(S R_{5}\right)$ separate the temporal and action parts from the "belief" part, which contains, in general, duplication of formulas with belief modalities. Despite this duplication, using the contraction rules and two sorts of marks, we can exclude loops for induction-free $R$-sequents (see Section 6) in derivations.

(b) Introducing two sorts of marks in simple separation rules $\left(S R_{l}^{*}\right), 1 \leqslant l \leqslant 3$, and using marked contraction rules we can also exclude loops. In this case we get more complex derivations. The separation rules $\left(S R_{l}\right), 1 \leqslant l \leqslant 5$, allow us to get more simple derivations but require more sophisticated analysis. 


\section{Decision Procedure for Induction-free $R$-sequents}

An $R$-sequent $S$ is an induction-free ( $I F R$-sequent) if $S$ does not contain positive occurrences of operators "unless" and "star". The decision procedure for $I F R$-sequents is realized by means of calculus for the induction-free temporal logic of belief and action (IFTBA).

DEFINITION 6 (calculus $I F T B A$ ). A calculus $I F T B A$ is obtained from the calculus $G W$ by adding the separation rules $\left(S R_{l}\right)(l \in\{1,2,3,4,5\})$ and the reduction rules, except for the rules $(\rightarrow Q \mathcal{W})$ and $(\rightarrow *)$.

Using induction on the height of derivation, we can show that all the reduction rules of the calculus IFTBA are invertible.

The separation rules $\left(S R_{l}\right)(l \in\{1,2,3,4,5\})$ are not simply invertible, but they are disjunctively invertible.

Using induction on the height of derivation, we can prove the following

Lemma 1. Let $S$ be a conclusion of the rules $\left(S R_{l}\right)(l \in\{1,2,3,4,5\})$ and IFTBA $S$, then either there exists $i(1 \leqslant i \leqslant n)$ such that IFTBA $\vdash S_{1}$, or there exists $i$ $(1 \leqslant i \leqslant n)$ and $\rho \geqslant 1$ such that IFTBA $\vdash S_{2}$, or there exists $l(1 \leqslant l \leqslant p)$ and $\rho \geqslant 1$ such that IFTBA $\vdash S_{3}$, or there exists such $l(1 \leqslant l \leqslant p)$ and $\rho \geqslant 1$ such that IFTB $A \vdash S_{4}$, or there exists $k(1 \leqslant k \leqslant n)$ such that IFTBA $\vdash S_{5}$.

Because of duplication of belief modalities in the separation rules $\left(S R_{3}\right),\left(S R_{4}\right)$, $\left(S R_{5}\right)$ there is a possibility to generate infinitely bottom-up applications of these rules, in general. To stop this infinite process let us introduce a notion of belief final ( $b$-final) $R$-sequents which will be a stopping device for non-derivable $R$-sequents in the calculus IFTBA.

An $R$-sequent $S^{*}$ is $b$-final if $S^{*}$ consists of only atomic formulas and/or marked modalities.

The decision procedure for an $I F R$-sequent $S$ is realized by constructing the socalled ordered derivations in the calculus $I F T B A$.

Definition 7. An ordered derivation $D$ for induction-free $R$-sequents consists of several horizontal levels. Each level consists of bottom-up applications of reduction rules. At each level, where a set consisting of only reduced-primary $R$-sequents is received, all possible bottom-up applications of the separation rules $\left(S R_{i}\right), i \in\{1,2,3,4,5\}$ to every reduced-primary $R$-sequent are realized. Each bottom-up application of the separation rules $\left(S R_{i}\right)(i \in\{1,2,3,4,5\})$ provides a possibility to construct a different (in general) ordered derivation $D_{k}(k \geqslant 1)$

The ordered derivation $D_{k}$ is a successful one, if each leaf of $D_{k}$ ends with a logical axiom. Let all possible ordered derivations $D_{k}$ be such that in each $D_{k}$ there exists a branch having either such a leaf that a sequent in this leaf contains only atomic formulas and is not an axiom, or an induction-free $R$-sequent $S^{*}$ such that $S^{*}$ is a $b$-final $R$-sequent. In these cases, IFTBA $\nvdash S$ and derivation is an unsuccessful one. 
Theorem 1. Let $S$ be an induction-free $R$-sequent. Then one can automatically construct a successful or unsuccessful ordered derivation $D$ of the R-sequent $S$ in IFTBA such that $D$ always terminates.

Proof. The automatic way of construction of an ordered derivation $D$ and correctness (i.e., preservation of derivability) follows from invertibility of the rules of the calculus IFTBA; termination follows from finiteness of the generated subformulas in $D$ and from the shape of separation rules $\left(S R_{l}\right), 1 \leqslant l \leqslant 5$.

EXAMPLE 5. (a) Let $S=\operatorname{Bel}(i) A \rightarrow$, where $A=\left(\left[\alpha_{1}\right] P \wedge \neg\left[\alpha_{1}\right] Q\right)$. Bottom-up applying $\left(S R_{5}\right)$ (and then $(\wedge \rightarrow),(\neg \rightarrow)$ ) to $S$, we get (using that $A$ does not contain belief modalities) an $R$-sequent $S_{1}=\left[\alpha_{1}\right] P \rightarrow\left[\alpha_{1}\right] Q$. To $S_{1}$ we can bottom-up apply only $\left(S R_{2}\right)$ and get an $R$-sequent $P \rightarrow Q$, which is not an axiom. Therefore $I F T B A \nvdash S$.

(b) Let $S=\operatorname{Bel}(i) A \rightarrow$, where $A=\left(\left[\alpha_{1}\right] P \wedge \neg \operatorname{Bel}(i) Q\right)$. Bottom-up applying $\left(S R_{5}\right)$ (and then $\left.(\wedge \rightarrow),(\neg \rightarrow)\right)$ we get an $R$-sequent $S_{1}=\operatorname{Bel}^{*}(i) A,\left[\alpha_{1}\right] P \rightarrow$ $\operatorname{Bel}(i) Q$. Since $\left[\beta_{k}\right] \nabla=\varnothing$ and $Q$ does not contain a negative occurrence of belief modality, we can bottom-up apply only $\left(S R_{4}\right)$ (and then $(\wedge \rightarrow),(\neg \rightarrow)$ and implicitly the marked contraction rules) and get an $R$-sequent $S_{2}=\operatorname{Bel}^{*}(i) A,\left[\alpha_{1}\right]^{*} P \rightarrow$ $\mathrm{Bel}^{+}(i) Q, Q$. Therefore $S_{2}$ is $b$-final $R$-sequent and $I F T B A \nvdash S$.

EXAmple 6. Let $S^{*}=\forall\left(P_{1} \mathcal{W}\left(\left[\alpha^{*}\right] P_{2} \vee \operatorname{Bel}(i) P_{3}\right) \rightarrow \Delta\right.$, where $\Delta=P_{1},[\alpha] P_{2}$, $\operatorname{Bel}(i)\left(\operatorname{Bel}(i)\left(P_{3} \vee P_{4}\right)\right)$. Then $S^{*}$ is a primary induction-free $R$-sequent. Let us construct an ordered derivation. First we reduce the sequent $S^{*}$ to a set of reduced primary sequents. Since $S^{*}$ does not contain either positive occurrences of "unless" or "next", instead of bottom-up application of $(Q \mathcal{W} \rightarrow)$ we can bottom-up apply $\left(\mathcal{W}_{0} \rightarrow\right)$ to $S^{*}$ and get the $R$-sequent $S_{0}^{*}=P_{1} \vee\left(\left[\alpha^{*}\right] P_{2} \vee \operatorname{Bel}(i) P_{3}\right) \rightarrow \Delta$. Bottom-up applying the rules $(\vee \rightarrow),(* \rightarrow)$, from the sequent $S_{0}^{*}$, we get three reduced-primary $R$-sequents:

$S_{1}^{*}=P_{1} \rightarrow \Delta ; S_{2}^{*}=P_{2},[\alpha]\left[\alpha^{*}\right] P_{2} \rightarrow \Delta ; S_{3}^{*}=\operatorname{Bel}(i) P_{3} \rightarrow \Delta$.

The sequent $S_{1}^{*}$ is an axiom.

Let us consider the $R$-sequent $S_{2}^{*}$. We can bottom-up apply the separation rules $\left(S R_{2}\right)$ or $\left(S R_{4}\right)$ to the sequent $S_{2}^{*}$. Let us try to bottom-up apply the rule $\left(S R_{4}\right)$ to $S_{2}^{*}$. Then we get a reduced-primary $R$-sequent $S_{21}^{*}=\rightarrow \operatorname{Bel}(i)\left(P_{3} \vee P_{4}\right)$. We can bottom-up apply the rule $\left(S R_{4}\right)$ to the sequent $S_{21}^{*}$ and, after applying bottom-up the rule $(\rightarrow \vee)$, we get a sequent $\rightarrow P_{3} P_{4}$ which is not an axiom. Now let us bottom-up apply the rule $\left(S R_{2}\right)$ to $S_{2}^{*}$ and get an $R$-sequent $S_{22}^{*}=\left[\alpha^{*}\right] P_{2} \rightarrow P_{2}$. Since $S_{22}^{*}$ does not contain positive occurrences either of $*$ or action constants, bottom-up applying $\left(*_{0} \rightarrow\right)$ to $S_{22}^{*}$ we get an axiom $P_{2} \rightarrow P_{2}$.

Now let us consider the $R$-sequent $S_{3}^{*}$. We can bottom-up apply the separation rules $\left(S R_{2}\right)$ or $\left(S R_{4}\right)$ to the sequent $S_{3}^{*}$. Bottom-up applying $\left(S R_{2}\right)$ to $S_{3}^{*}$, we get the sequent $\rightarrow P_{2}$, which is not an axiom. Bottom-up applying $\left(S R_{4}\right)$ to $S_{3}^{*}$, we get the reduced primary $R$-sequent $S_{31}^{*}=P_{3}, \mathrm{Bel}^{*}(i) P_{3} \rightarrow \operatorname{Bel}(i)\left(P_{3} \vee P_{4}\right)$. We can bottom-up apply the rule $\left(S R_{4}\right)$ to the sequent $S_{31}^{*}$ and get the sequent $P_{3} \rightarrow P_{3} \vee P_{4}$, and after bottom-up applying the rule $(\rightarrow \vee)$, we get an axiom $P_{3} \rightarrow P_{3}, P_{4}$. 
Therefore, for the $I F R$-sequent $S^{*}$, we have constructed a successful ordered derivation (in the calculus $I F T B A$ ). Hence $I F T B A \vdash S^{*}$.

\section{Decision Algorithm for Arbitrary $R$-sequents}

In this section, we consider arbitrary $R$-sequents, i.e., sequents with positive occurrences of "unless" and "star" operators. As indicated in Section 3, positive occurrences of these operators allow us to formulate induction-like properties of temporal and action parts of $T L B A$. This fact necessitates a departure from ordinary Gentzen-like calculi. The basic positive closure axiom $\Gamma, A \rightarrow \Delta, A$ of the calculi, described in the previous sections, is not sufficient for an arbitrary $R$-sequent. The positive branch closure now requires an inspection of $R$-sequents other than a logical axiom in a leaf of derivation. This idea was raised in (Wolper, 1985) and realized for the tableau-like temporal calculus. Wolper's idea is wide used in the works on the temporal resolution calculus of Liverpool school (see, e.g., Fisher et al., 2001). Wolper's idea was rediscovered in (Pliuškevičius, 1993) for the sequent-like temporal calculus with separation rules, and subsequently used in (Pliuškevičius, 1994; 1996; 1998) and (Pliuškevičius and Pliuškevičienè, 2003).

So, along with the logical axioms, we introduce notions of temporal saturated ( $t$ saturated) and action saturated ( $a$-saturated) $R$-sequents that play the role of non-logical axioms. The decision algorithm for an arbitrary $R$-sequent is realized by means of a temporal calculus for belief and action $(T B A)$.

Let $D$ be a derivation in some calculus and $(i)$ be a branch in $D$. The $R$-sequent $S^{*}=\Gamma \rightarrow \Delta$ from the branch $(i)$ is a saturated $R$-sequent if, in the branch $(i)$ above $S^{*}$, there exists an $R$-sequent of the shape $S^{* *}=\Gamma, \Pi \rightarrow \Delta, \Theta$, in a special case, $S^{*}=S^{* *}$.

A saturated $R$-sequent $S^{*}$ is $t$-saturated if $S^{*}=\Gamma \rightarrow \Delta, Q(A \mathcal{W} B)$, and a saturated $R$-sequent $S^{*}$ is a-saturated if $S^{*}=\Gamma \rightarrow \Delta,\left[\alpha^{*}\right] A$. These sequents will be used as non-logical axioms.

DEFINITION 8 (calculus $T B A$ ). A calculus $T B A$ is obtained from the calculus $I F T B A$ by adding: (1) non-logical axioms of the shape $\Gamma \rightarrow \Delta, Q(A \mathcal{W} B)$ or $\Gamma \rightarrow \Delta,\left[\alpha^{*}\right] A$ and (2) the reduction rules $(\rightarrow Q \mathcal{W}),(\rightarrow *)$.

We can present the decision procedure for an arbitrary $R$-sequent in the same way as in the case of induction-free $R$-sequents. Namely, we construct ordered derivations in the same manner, as described in the previous section. But there is a new substantial point: along with the logical axioms there are non-logical axioms. If there exists an ordered derivation $D$ of $R$-sequent $S$ such that in a leaf of each branch $(i)$ of $D$ there is either a logical axiom, or a non-logical axiom, then in both these cases $T B A \vdash S$ (positive criterion of termination of the procedure). If in all the possible ordered derivations $D_{k}$ of an $R$-sequent $S$ there exists a branch having an induction-free $R$-sequent $S^{+}$such that $I F T B A \nvdash S^{+}$, then $T B A \nvdash S$ (negative criterion of termination of the decision procedure). 
To justify the presented decision procedure, we must found: (1) termination of the procedure and (2) disjunctive invertibility of the separation rules $\left(S R_{i}\right)(i \in\{1,2,3,4,5\})$ in $T B A$. The foundation of the negative criterion of termination of the procedure follows from the regularity condition of $R$-sequents and decidability of induction-free $R$ sequents. The positive criterion of termination will be founded by means of finiteness of the so-called $R$-subformulas of primary $R$-sequents which are generated during the construction of an ordered derivation. Let us define the precise notion of $R$-subformulas of an $R$-sequent. This notion corresponds to Fischer-Ladner closure (see, e.g., Emerson, 1990; Harel et al., 2000).

DEFINITION 9 ( $R$-subformulas). Let $S$ be a primary $R$-sequent and $C$ be a formula entering $S$. A set of $R$-subformulas of $C$ from $S$ is denoted as $R S u b(C)$ and defined inductively.

1. $R S u b(P)=\varnothing$, where $P$ is a propositional symbol.

2. $R S u b(Q \bigcirc A)=R S u b(A)$, where $Q \in\{\forall, \exists\}$.

3. $R \operatorname{Sub}(\neg A)=R S u b(A)$.

4. $R \operatorname{Rub}(A \odot B)=\{R S u b(A)\} \cup\{R S u b(B)\}(\odot \in\{\supset, \wedge, \vee\})$.

5. $R \operatorname{Sub}(\operatorname{Bel}(i) A)=\operatorname{Bel}(i) A \cup\{R S u b(A)\}$.

6. $R S u b(Q(A \mathcal{W} B))=Q \bigcirc Q(A \mathcal{W} B) \cup\{R S u b(A)\} \cup\{R S u b(B)\} ; Q \in\{\forall, \exists\}$.

7. $R \operatorname{Sub}\left(\left[\alpha_{i}\right] A\right)=R \operatorname{Rub}(A)$, where $\alpha_{i}$ is an agent constant.

8. $R \operatorname{Sub}\left(\left[\alpha^{*}\right] A\right)=[\alpha]\left[\alpha^{*}\right] A \cup\{R S u b(A)\}$.

9. $R S u b[\alpha \circ \beta] A=R S u b([\alpha][\beta] A)$.

10. $R S u b[\alpha \cup \beta] A=\{R S u b([\alpha] A)\} \cup\{R S u b([\beta] A)\}$.

11. $R S u b([P ?] A)=R S u b(A)$.

A set of $R$-subformulas of the $R$-sequent $S=A_{1}, \ldots, A_{n} \rightarrow A_{n+1}, \ldots, A_{n+m}$ (or a set of formulas $S=\left\{A_{1}, \ldots, A_{n+m}\right\}$ ) is denoted by $R S u b(S)$ and defined as $R S u b(S)=\cup_{i=1}^{n+m} R S u b\left(A_{i}\right)$.

From the definition of $R S u b(S)$ we get that for each primary $R$-sequent $S$, the set $R S u b(S)$ is finite. Let $S$ be a given primary $R$-sequent, $D$ be an ordered derivation of $S$, and $S_{i}$ be a primary $R$-sequent from $D$. Then, from the shape of the rules of the calculus $T B A$, the description of an ordered derivation, and from the definition of $R S u b(S)$ we get that $R S u b\left(S_{i}\right) \subseteq R S u b(S)$. Therefore nothing new can be obtained in constructing an ordered derivation of an $R$-sequent. Hence, if an ordered derivation $D$ does not contain an induction-free $R$-sequent $S_{i}$ such that $I F T A B \nvdash S_{i}$, then in a leaf of each branch $(i)$ of $D$ there is either a logical axiom or a non-logical axiom.

Hence the termination of the positive criterion of the decision procedure is justified. To justify the disjunctive invertibility of the separation rules $\left(S R_{i}\right)(i \in\{1,2,3,4,5\})$ an infinitary calculus $T B A_{\omega}$ is introduced.

Definition 10 (calculus $T B A_{\omega}$ ). A calculus $T B A_{\omega}$ is obtained from the calculus $T B A$ by means of: (1) elimination of non-logical axioms and (2) replacing the rules $(\rightarrow Q \mathcal{W}),(\rightarrow *)$ by the rules $\left(\rightarrow Q \mathcal{W}_{\omega}\right)$ and $\left(\rightarrow *_{\omega}\right)$ (see Section 3$)$. 
Using double induction on $\langle n, h\rangle$, where $n$ is the number of positive occurrences of $\mathcal{W}$ and $*, h$ is the height of derivation, analogously as in (Pliuškevičius, 1998), we can justify the disjunctive invertibility (see Lemma 1 ) of the separation rules $\left(S R_{i}\right)$ ( $i \in$ $\{1,2,3,4,5\})$ in $T B A_{\omega}$. To prove that $T B A_{\omega}$ and $T B A$ are equivalent for the class of $R$-sequents, let us introduce the invariant calculus $I N T B A$, which is obtained from the calculus $T B A$, replacing the non-logical axiom by the following invariant rules:

$$
\begin{aligned}
& \frac{\Gamma \rightarrow \Delta, I ; I \rightarrow B, Q \bigcirc I ; I \rightarrow A, B}{\Gamma \rightarrow \Delta, Q(A \mathcal{W} B)}\left(\rightarrow Q \mathcal{W}^{\prime}\right), \\
& \frac{\Gamma \rightarrow \Delta, I ; I \rightarrow[\alpha] I ; I \rightarrow A}{\Gamma \rightarrow \Delta,\left[\alpha^{*}\right] A}\left(\rightarrow *^{\prime}\right),
\end{aligned}
$$

where the formula $I$ is called an invariant formula and is constructed automatically, using non-logical axioms (analogously as in (Pliuškevičius, 1994)). Analogously as in (Pliuškevičius, 1998), we can show that the calculi $T B A, I N T B A$, and $T B A_{\omega}$ are equivalent for the class of $R$-sequents. Therefore the rules $\left(S R_{i}\right)(i \in\{1,2,3,4,5\})$ are, also, disjunctively invertible in the calculus $T B A$. Hence the justification of the main points of the presented decision algorithm for an arbitrary $R$-sequent is complete and we can get the following

Theorem 2. Let $S$ be a non-induction-free $R$-sequent. Then one can automatically construct a successful or unsuccessful ordered derivation $D$ of the R-sequent $S$ in TBA such that D always terminates.

Proof. The automatic way of construction of an ordered derivation $D$ and correctness (i.e., preservation of derivability) follow from invertibility of the rules of $T B A$; the termination follows from finiteness of $R S u b(S)$.

REMARK 4. (a) To make the presented decision procedure more effective some subsumption rules can be introduced with the help of which non-essential $R$-sequents can be eliminated.

(b) The complexity of the presented algorithm (as in a temporal logic (see, e.g., Emerson, 1990) and in PDL (see, e.g., Harel et al., 2000)) is PSRACE-complete, i.e., during the construction of an ordered derivation we generate an $R$-sequence the length of which can be restricted by some polynomial depending on occurrences of the operators $\mathcal{W}$ ("unless") and * ("star").

EXAMPLE 7. Let $S=\forall(A \mathcal{W}(B \vee C)) \rightarrow \exists(A \mathcal{W} B), \exists(A \mathcal{W} C)$. Let us construct an ordered derivation of $S$ in the calculus $T B A$. First we try to reduce the sequent $S$ to a set of reduced primary sequents. It is easy to verify that during this process and bottomup applying the reduction rules, we get the $R$-sequent of the shape $S_{1}=A \vee(B \vee C)$, $\Gamma \rightarrow A, B, C, \Delta$ and the reduced primary $R$-sequent $S_{2}=A \vee(B \vee C), \forall \bigcirc(\forall(A \mathcal{W}(B \vee$ $C), \forall \bigcirc \neg \exists(A \mathcal{W} B)), \forall \bigcirc \neg \exists(A \mathcal{W} C)) \rightarrow B$. From $G W \vdash A \vee(B \vee C), \Gamma \rightarrow A, B, C$, $\Delta$ follows that $T B A \vdash S_{1}$. Let us consider the $R$-sequent $S_{2}$. We can bottom-up apply 
only the rule $\left(S R_{1}\right)$ to the sequent $S_{2}$ and get an $R$-sequent $S_{21}=\forall(A \mathcal{W}(B \vee C))$, $\neg \exists(A \mathcal{W} B)), \neg \exists(A \mathcal{W} C) \rightarrow$. Bottom-up applying the rule $(\neg \rightarrow)$ to $S_{21}$, we get an $R$-sequent $S^{*}=\forall(A \mathcal{W}(B \vee C)) \rightarrow \exists(A \mathcal{W} B), \exists(A \mathcal{W} C)$. Since $S^{*}=S, S$ is a $t$-saturated one, i.e., a non-logical axiom. Therefore we have constructed a successful ordered derivation having logical axioms and one non-logical axiom. Hence $T B A \vdash S$.

\section{Conclusions and Further Investigations}

In the paper, the decision algorithm for a restricted temporal logic of belief and actions is presented $(T L B A)$. The considered logic allows us to express informational and dynamic properties of rational agents (see Sections 2, 3). The regularity condition (see Section 3) allows us: 1) to separate the decision procedure for induction-free sequents and 2) to use only logical axioms in leaves of derivations for these sequents (see Section 5). The main technical tool of the presented decision algorithm is the separation rules $\left(S R_{i}\right)$ $(i \in\{1,2,3,4,5\})$ which incorporate traditional rules for the "next" operator, multimodal belief modalities, agent constants, and reflect the properties of belief modalities.

Unfortunately, the considered $T L B A$ does not contain, just like $B D I, K A R O$ and $L O R A$ logics, any tools for interaction between different agents and for action interactions. So, in future investigations, we are going to consider agent-like logics with interacting beliefs and actions and containing tools for communications. Another interesting and important investigation is related with the consideration of some fragments of first-order agent-based logics, extension results in (Pliuškevičius, 2000; Pliuškevičius and Pliuškevičienè, 2003), and with consideration of some fragments of the first-order agent-like logic LORA (Wooldridge, 2000).

\section{Acknowledgement}

The authors thank an anonymous referee for remarks.

\section{References}

Clarke, E., O. Grumberg and D. Peled (2000). Model Checking. MIT Press.

Emerson, E.A. (1990). Temporal and modal logic. In J. van Leeuwen (Ed.), Handbook of Theoretical Computer Science, Formal Models and Semantics, Vol. B. Elsevier Science Publ., Amsterdam. pp. 996-1072.

Fisher, M., and M. Wooldridge (1997). On the formal specification and verification of multi-agent systems. Intern. Journal of Cooperative Information Systems, 6(1), 37-65.

Fisher, M., C. Dixon and M. Peim (2001). Clausal temporal resolution. ACM Transactions on Computational Logic, 2(1), 12-56.

Fitting, M. (1993). Basic modal logic. In D.M. Gabbay, C.J. Hogger, J.A. Robinson (Eds.), Handbook for Logic in Artificial Intelligence and Logic Programming. Logical Foundations, Vol. 1. Claredon Press, Oxford. pp. 365-447.

Harel, D., D. Kozen, J. Tiuryn (2000). Dynamic Logic. MIT Press.

Hudelmaier, J. (1996). A contraction-free sequent calculus for S4. In H. Wansing (Ed.), Proof Theory for Modal Logic. Kluwer Academic Publishers, Dordrechts, Boston/London. pp. 3-16. 
Gentzen, G. (1934-35). Untersuchungen über das logische Schliessen. I, II. Mathematische Zeitschrift, 39 176-210, 405-431.

Kawai, H. (1987). Sequential calculus for a first-order infinitary temporal logic. Zeitchr. für Math. Logic and Grundlagen der Math., 33, 423-432.

Meyer, J.J.Ch., van der Hoek (1995). Epistemic Logic for AI and Computer Science. Cambridge University Press, Cambridge.

Nide, N., S. Takata (2002). Deduction systems for BDI logics using sequent calculus. In Proc. of AAMAS'02. pp. 928-935.

Pliuškevičius, R. (1991). Investigation of finitary calculus for a discrete linear time logic by means of infinitary calculus. Lecture Notes in Computer Science, 502, 504-528.

Pliuškevičius, R. (1993). On saturation principle for a linear temporal logic. Lecture Notes in Computer Science, 713, 289-300.

Pliuškevičius, R. (1994). The saturated tableaux for linear miniscoped Horn-like temporal logic. Journal of Automated Reasoning, 13, 51-67.

Pliuškevičius, R. (1996). Similarity saturation for first order linear temporal logic with UNLESS. Lecture Notes in Artificial Intelligence, 1126, 320-336.

Pliuškevičius, R. (1998). Replacement of induction by similarity saturation in a first-order linear temporal logic Journal of Applied Non-classical Logics, 8(1-2), 141-169.

Pliuškevičius, R. (2000). On an $\omega$-decidable deductive procedure for non-Horn sequents of a restricted FTL. Lecture Notes in Artificial Intelligence, 1861, 523-537.

Pliuškevičius, R. (2001). Deduction-based decision procedure for a clausal miniscoped fragment of FTL. Lecture Notes in Artificial Intelligence, 2083, 107-120.

Pliuškevičius, R., and A. Pliuškevičienė (2003). Decision procedure for a fragment of FTL with equality. In C. Mayer, F. Pirri (Eds.), TABLEAUX 2003, Position papers and tutorials. Technical report RT-DIA-80-2003 Dipartimento di Informatica e Automazione Universita degli Studi di Roma TreM. pp. 55-67.

Rao, A.S., M. Georgeff (1991). Modeling rational agents within a BDI-architecture. In J.F. Allen, R. Fikes, E. Sandewall (Eds.), Knowledge Representation and Reasoning. Morgan Kaufman, San Mateo, Ca. pp. 473 484

Rao, A.S., M.P. Georgeff (1998). Decision procedures for BDI logics. Journal of Logic and Computation, 8(3), 292-343.

Shwarts, G.F. (1989). Gentzen style systems for K45 and K45D. Lecture Notes in Computer Science, 363, $245-256$.

Wang, H. (1964). A Survey of Mathematical Logic, Chapter 9. Science Press, Peking.

Wolper, P. (1985). The tableaux method for temporal logic: an overview. Logique et Analyse, 28, 119-136.

Wooldridge, M., N.R. Jennings (1995). Intelligent agents: Theory and practice. The Knowledge Engineering Review, 10(2), 563-594.

Wooldridge, M. (2000). Reasoning about Rational Agents. The MIT Press.

Wooldridge, M., C. Dixon, M. Fisher (1998). A tableau-based proof method for temporal logics of knowledge and belief. Journal of Applied Non-classical Logics, 6(3), 225-258. 
R. Pliuškevičius is a hab. doctor of mathematical sciences, associated professor. He is a head of Mathematical Logic Department at the Institute of Mathematics and Informatics. He is a member of Lithuanian Mathematical Society, American Mathematical Society, European Association for Theoretical Computer Science, European Association for Computer Science Logic. His main research interests include computer-aided calculi for temporal logics; applications of temporal logics for verification and specification of reactive systems; agent-based logics, temporal logics, decision procedures.

A. Pliuškevičienè is a doctor of mathematical sciences, associated professor. She is a senior scientific researcher of Mathematical Logic Department at the Institute of Mathematics and Informatics. The field of research - proof theory of classical and non-classical logics, agent-based logics, temporal logics, decision procedures, modal logics.

\section{Išsprendžiamoji procedūra tikẻjimo ir veiksmụ laiko logikai} Regimantas PLIUŠKEVIČIUS, Aida PLIUŠKEVIČIENĖ

Pateikiama išsprendžiamoji procedūra kompiuteriniu agentu logikai, ijungiančiai laiko logiką $C T L$, multimodalumo logiką $K D 45_{n}$ ir teiginių dinaminę logiką. Šios logikos aprašo informacines ir dinamines kompiuteriniu agentu savybes. 